\title{
超额光谱及其研究进展
}

\author{
周 瑜 ${ }^{1}$ 徐 静 $^{1} \quad$ 王楠楠 $^{2}$ 尉志武1,* \\ ('清华大学化学系, 生命有机磷化学及化学生物学教育部重点实验室, 北京 100084 ; \\ 2 国防科学技术大学航天科学与工程学院, 新型陶瓷纤维及其复合材料重点实验室, 长沙 410073)
}

\begin{abstract}
摘要: 超额光谱是借助于热力学中超额函数的概念提出的, 是经典热力学函数的补充, 它可以提供丰富的分 子间相互作用的信息。本文首先介绍了超额光谱的概念和测定方法。然后介绍了超额光谱的用途：提高谱图 的表观分辨率、判断体系的非理想性、判断分子间选择性相互作用的优先性、判断二态性以及稳定缔合体的 存在和提供分子电荷分布的信息等。其后介绍了超额光谱方法学的若干新进展：超额拉曼光谱、超额偏摩尔 红外光谱和固体假二元超额光谱。最后, 介绍了超额光谱在几个方面的最新应用: 离子液体和分子溶剂体系 中的氢键、苯衍生物和二甲基亚砜(DMSO)中的卤键、无机阴阳离子和水的相互作用。综上, 超额光谱可以 帮助我们得到更加丰富的分子间相互作用的信息，为该领域的研究打开了一扇新窗户。
\end{abstract}

关键词: 超额光谱; 衰减全反射红外光谱; 氢键; 卤键; 分子间相互作用; 聚集体; 物种指认 中图分类号：0645; 0642

\section{Excess Spectroscopy: Concept and Applications}

\author{
ZHOU $\mathrm{Yu}^{1} \quad \mathrm{XU}$ Jing $^{1} \quad$ WANG Nan-Nan ${ }^{2} \quad \mathrm{YU}$ Zhi-Wu, ${ }^{1, *}$ \\ $\left({ }^{1}\right.$ Key Laboratory of Bioorganic Phosphorous Chemistry and Chemical Biology (Ministry of Education), Department of Chemistry, \\ Tsinghua University, Beijing 100084, P. R. China; ${ }^{2}$ Key Laboratory of Science and Technology on Advanced Ceramic Fibers and \\ Composites, College of Aerospace Science and Engineering, National University of Defense Technology,
}

Changsha 410073, P. R. China)

\begin{abstract}
Excess spectroscopy was proposed following the idea of excess thermodynamic functions. It is complementary to classical excess functions because it provides rich information on molecular interactions. In this review, we introduce in detail the concept of excess spectroscopy and the measurement of excess spectra for the case of infrared spectroscopy. We then describe the merits of using excess spectroscopy to enhance apparent spectral resolution, judge the non-ideality of mixtures, determine the selectivity of molecular interactions, identify distinct species or clusters in solutions, and provide information related to charge distributions in molecules. Following this, we review the progress in methodology where excess spectroscopy is extended to partial molar excess spectroscopy and Raman spectroscopy. The extension of binary mixtures to pseudo binary mixtures and/or liquid samples to solid samples is also described. Finally, we discuss several recent applications of excess spectroscopy in the study of hydrogen-bonding interactions in ionic liquidmolecular solvent systems, halogen-bonding interactions in benzene derivative-dimethylsulfoxide (DMSO) mixtures, and interactions between inorganic cations/anions and water molecules. Clearly, excess spectroscopy has opened a new window through which we can view rich information about molecular interactions.
\end{abstract}

Received: October 19, 2015; Revised: November 23, 2015; Published on Web: November 24, 2015.

*Corresponding author. Email: yuzhw@mail.tsinghua.edu.cn; Tel: +86-10-62792492.

The project was supported by the National Natural Science Foundation of China (21133009, 21473099).

国家自然科学基金(21133009, 21473099)资助项目

CEditorial office of Acta Physico-Chimica Sinica 
Key Words: Excess spectra; ATR-IR; Hydrogen bond; Halogen bond; Molecular interaction; Cluster; Species identification

\section{1 引言}

用实验或者理论的方法研究溶液结构是物理 化学最艰巨的任务之一。液体的分子间相互作用 很强, 分子坐标不确定, 这使得研究溶液结构非 常困难。然而, 多数化学反应以及提取、纯化和 萃取分离等过程都是在溶液中进行的, 因此深入 认识液体物质的物理化学性质具有非常重要的意 义。结构决定性质, 深入研究溶液的结构是认识 其物理化学性质的基础。

溶液中一个分子与周围分子间的关系可以分 为两种可能的情况。其一, 不存在特殊的相互作 用, 因此没有优势配对和接触方位关系, 随机运 动使得配对寿命很短; 其二, 存在特殊的相互作 用，因此有优势的配对关系，配对的寿命也比较 长。后一种情况中一般存在着氢键、卤键、配位 键等强分子间相互作用, 即分子间发生了缔合现 象。探究溶液的缔合现象意义重大, 它是发展溶 液理论的基础。若溶液中形成了大的、稳定的缔 合体(或聚集体)例如超分子结构, 可以通过电镜等 手段进行表征 ${ }^{2,3}$, 若是溶液中形成的缔合体比较 小, 而且不稳定, 则很难通过实验手段进行表 征 $^{4-6}$ 。X光散射、质谱、光谱等技术都只能从某一 个侧面获得部分缔合体的信息, 与人们的期望值 还相差甚远。X光散射技术需要缔合体与周围溶剂 有较大的电子密度差才能获得低分辨率的结构信 息, 径向分布函数所能提供的信息也非常有限; 质谱法可以给出明确的缔合度(聚集度)信息, 但要 求缔合体有高的稳定性; 光谱法快速方便, 能解 决一些问题，但其广泛的应用受制于分辨率不高 和谱峰的指认困难。发展有效的检测方法和建立 合理的液体模型是溶液结构研究中亟待解决的问 题。

1935年Scatchard和Hamer ${ }^{7}$ 提出了超额自由能的 概念, 这是最早的超额热力学函数。常用的热力 学超额函数有超额体积、超额焓、超额熵、超额 自由能等, 我们实验室曾开展过一些相关的研究 工作 ${ }^{8-11}$ 。超额函数反映的是溶液的宏观性质, 而分 子光谱可以从分子层次上揭示液体混合物的内部 结构信息。所以，如果能把超额函数和分子光谱 结合起来研究, 就有可能更全面地认识分子间相
互作用的基本规律。2006年本课题组借助化学热 力学中超额函数的思想, 将超额的概念引入到光 谱学, 提出了宏观溶液体系的超额光谱概念, 并 将其应用到了红外光谱分析中 ${ }^{12,13}$ 。所谓超额红外 光谱是指相同条件下, 实际溶液的红外光谱与理 想溶液的红外光谱的差值, 以超额摩尔吸光度的 形式表示如下:

$$
\varepsilon^{\mathrm{E}}=\varepsilon-\varepsilon^{\text {ideal }}
$$

式中 $\varepsilon$ 表示实际溶液中某一波数处的摩尔吸光度,

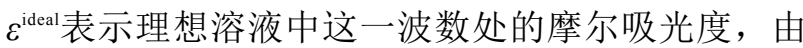
两组分摩尔吸光度按摩尔分数的线性加和得到。 $\varepsilon^{\mathrm{E}}$ 的意义是：该波数下，实际溶液的摩尔吸光度对 理想溶液摩尔吸光度的偏离。超额摩尔吸光度能 够直观地体现体系中的分子间相互作用对基团振 动情况的影响。通过研究混合溶液体系的超额光 谱, 可以在分子或亚分子层次上研究混合溶液中 分子间的相互作用，这为分子间相互作用的研 究、为认识分子识别的本质开辟了一条新的思 路，有望在分子及亚分子层次回答我们长期关 注、但一直不能很好回答的分子间相互作用的问 题。

超额光谱的概念经过 10 年的发展已经越来越 完善, 逐渐成为一种研究溶液结构的有效方法。 在方法学上, 超额光谱的概念被逐渐发展并延伸 到其它的光谱中，例如拉曼光谱 ${ }^{14,15}$; 在实际应用 上, 多个课题组用超额光谱的方法, 在不同领域 做了大量优秀的工作，使得超额光谱的应用被逐 渐扩宽，成为研究分子间相互作用的重要方法。 本文主要介绍超额光谱的概念和数据处理以及超 额光谱在方法学上的发展和应用上的进展。

\section{2 超额光谱的理论计算公式和实验方法}

2.1 超额红外光谱理论计算公式

以恒温下二组分溶液体系为例, 说明超额红 外光谱的计算公式。

对于纯物质的红外光谱, 其吸光度可以用 Beer-Lambert定律的形式表示

$$
A^{*}=\varepsilon^{*} d C^{*}
$$

式中, $\varepsilon^{*}$ 表示纯物质在某一波数处的摩尔吸光度或 
摩尔吸光系数 $\left(\mathrm{L} \cdot \mathrm{mol}^{-1} \cdot \mathrm{cm}^{-1}\right), d$ 表示光程 $(\mathrm{cm})$, $C^{*}$ 表示纯物质的摩尔浓度 $\left(\mathrm{mol} \cdot \mathrm{L}^{-1}\right)$, 对于混合溶液 体系, 某一波数处的吸光度也采用这一形式来表 示:

$$
A=\varepsilon d C
$$

式中, $C$ 为体系中所有组分的总浓度。对于二组分 溶液体系, 公式(3)可以表示为:

$$
A=\varepsilon d\left(C_{1}+C_{2}\right)
$$

式中, $C_{1}$ 和 $C_{2}$ 分别代表混合体系中组分 1 和组分 2 的 摩尔浓度 $\left(\mathrm{mol} \cdot \mathrm{L}^{-1}\right)$ 。需要强调的是, 对于实际溶 液, 某一波数处的摩尔吸光度 $\varepsilon$ 是溶液组成的函 数。

根据吸光度的加和性, 理想溶液的摩尔吸光 度可用纯组分的摩尔吸光度表示:

$$
\varepsilon^{\text {ideal }}=x_{1} \varepsilon_{1}^{*}+x_{2} \varepsilon_{2}^{*}
$$

式中, $x_{1}$ 和 $x_{2}$ 分别表示混合体系中组分 1 和组分 2 的 摩尔分数, $\varepsilon_{1}^{*}$ 和 $\varepsilon_{2}^{*}$ 分别表示这两个纯组分的摩尔吸 光度 $\left(\mathrm{L} \cdot \mathrm{mol}^{-1} \cdot \mathrm{cm}^{-1}\right)$, 可以通过测定纯物质的红外 光谱得到。

将公式(4)和(5)代入公式(1), 可以得到一定温 度下, 某一波数处超额摩尔吸光度的计算公式:

$$
\varepsilon^{\mathrm{E}}=\frac{A}{d\left(C_{1}+C_{2}\right)}-\left(x_{1} \varepsilon_{1}^{*}+x_{2} \varepsilon_{2}^{*}\right)
$$

由于 $\varepsilon^{*}$ 表示纯物质的摩尔吸光度, 因此, 在计算不 同浓度的超额红外光谱时, $\varepsilon_{1}^{*}$ 和 $\varepsilon_{2}^{*}$ 为定值。通过公 式(6)可以得到各波数对应的超额摩尔吸光度 $\left(\varepsilon^{\mathrm{E}}\right)$, 即超额红外光谱。

\section{2 实验方法}

\section{2 .1 化学药品和溶液配制方法}

超额光谱很灵敏, 因此光谱实验中对药品纯 度的要求比较高, 若样品纯度低, 需提前对样品 进行纯化。

配制样品时, 采用称重法确定各个组分物质
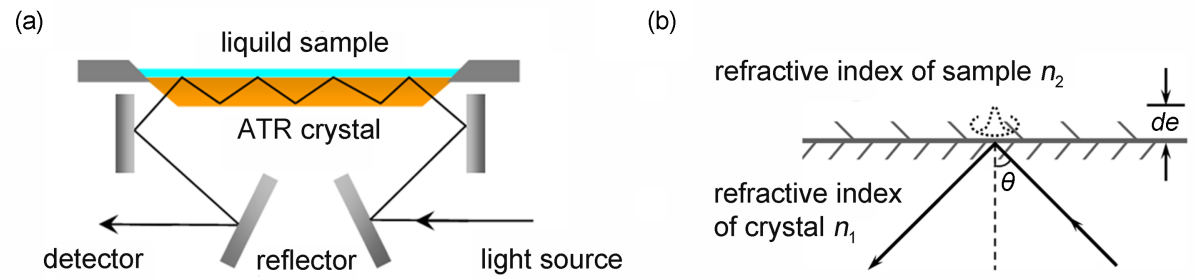

的量, 采用容量瓶确定样品总体积。由于超额体 积一般情况下很小, 可以假设体积具有加和性, 利用称重法和组分的密度估算溶液的总体积。更 精确地确定溶液体积的方法是通过实验建立溶液 的密度与摩尔分数间的关系, 进而通过密度计算 出溶液的体积。做超额红外光谱至少需要 3 个样 品, 纯组分 ( 2 个)和某一浓度的样品。若要做 $n$ 个浓 度的超额红外光谱则需要 $n+2$ 个样品。配制样品 应掌握的原则是：后加入易吸水、易挥发的组 分; 配制样品过程中, 要做到迅速, 并及时地做 好密封。

\section{2 .2 红外光谱实验}

为了保证定量分析的准确性, 红外光谱实验 中使用了水平的衰减全反射(ATR)附件, 其光路图 和工作原理如图1所示, 液体样品置于ATR附件上 方，红外光束在晶体内发生多次全反射后到达检 测器, 如图1(a)所示。红外光在晶体内表面发生全 反射时，在晶体外表面附近产生驻波，称为隐失 波 $^{16}$, 如图 1(b) 所示。当样品与晶体外表面接触 时, 在每个反射点隐失波都穿入样品, 从隐失波 衰减的能量可以得到样品的振动吸收信息。在使 用衰减全反射附件测定红外光谱时, 红外光对样 品的有效穿透深度(也就是光程)与入射光波长、入 射角、晶体折射率、样品折射率和反射次数有 关，其计算公式为 ${ }^{17}$ :

$$
\begin{aligned}
d e= & {\left[\frac{2 n_{21} \cos \theta}{\left(1-n_{21}^{2}\right)\left(\sin ^{2} \theta-n_{21}^{2}\right)^{\frac{1}{2}}}+\right.} \\
& \left.\frac{2 n_{21} \cos \theta\left(2 \sin ^{2} \theta-n_{21}^{2}\right)}{\left(\sin ^{2} \theta-n_{21}^{2}\right)^{\frac{1}{2}}\left(n_{21}^{4} \cos ^{2} \theta+\sin ^{2} \theta-n_{21}^{2}\right)}\right] \times \\
& \frac{N \lambda}{4 \pi n_{1}}
\end{aligned}
$$

式中, $\lambda$ 是入射光波长, $\theta$ 是入射角, $n_{1}$ 是晶体折射

图1 水平衰减全反射(ATR)附件光路示意图(a); 全反射界面隐失波示意图(b) 
率, $n_{21}$ 是样品折射率和晶体折射率的比值, $N$ 是反 射次数。

根据基团吸收的强弱, 可以选择不同的 ATR附件。在计算有效穿透深度时, 还需要知道 样品的折射率, 这可由阿贝折射仪测定。红外谱 图的处理过程为: 首先进行ATR校正, 然后将有 效穿透深度作为光程代入公式(6)计算超额摩尔吸 光度。

对于超额光谱的实验测量, 叔丁醇-四氯化碳 二元体系可作为标准体系, 用以检验仪器的正常 工作和数据的正确处理。

\section{3 超额红外光谱的用途}

\section{1 判断溶液的非理想性}

和其它超额函数一样, 超额光谱可以反映一 个体系的非理想性。理想溶液的超额光谱为零, 非理想溶液的超额光谱不为零。一个体系的超额 光谱越接近零, 说明这个体系越接近于理想体 系; 一个体系的超额光谱越大, 说明这个体系越 偏离于理想体系, 非理想性越大。与其它超额函 数不同, 超额光谱是从基团层次上反映体系的非 理想性。在一个分子中，不同基团的不同振动方 式的超额光谱的大小是不同的, 所以反映体系的 非理想性的能力是不一样的。一个基团的超额光 谱值越大, 说明它受到的影响越大, 体系的非理 想性越大。

图2是乙醇-甲醇、乙醇-四氯化碳以及乙醇-二 甲基亚砜三个体系的羟基伸缩振动区域的超额光 谱13。三个分图的超额光谱表明, 从图(a)到(c), 超 额峰越来越大, 表明非理想性越来越大。可以这
样来理解: 图2(a)中乙醇和甲醇在结构上非常相 似, 接近于理想体系, 所以其超额光谱的数值非 常小, 几乎等于零; 图2(b)中, 乙醇为极性分子, 而四氯化碳为非极性分子, 二者相差较大, 其超 额红外光谱的数值比较大; 图2(c)中, 二甲基亚砜 (DMSO) 和乙醇可以形成氢键, 该体系偏离理想体 系最大, 其超额值也最大。

\section{2 提高表观分辨率}

超额红外光谱可以提高光谱的表观分辨率。 在超额红外光谱中, 观测到的超额峰往往多于原 始光谱的峰。图3是叔丁醇-四氯化碳体系中羟基的 伸缩振动区域的红外光谱和超额红外光谱 ${ }^{13}$ 。由于 羟基间存在氢键作用, 所以叔丁醇分子是高度缔 合的, 以多种缔合形式存在。在图3中, 叔丁醇-四 氯化碳体系的红外光谱可以观察到一大一小两个 吸收峰, 而超额红外光谱中可以观测到四个超额 峰。很显然, 超额红外光谱提高了红外光谱的表 观分辨率。

对图3(b)中超额光谱的理解与指认是超额光谱 学的一个关键点。设想我们关注的羟基(及其相关 联的叔丁醇分子)在其纯态有若干种存在形式, 例 如单体、二聚体等等。为方便起见, 我们可以称 其为“物种”。每一“物种”对应于一个红外吸收峰, 实验所观测到的红外吸收峰(或吸收带)实际上是所 有可能物种吸收峰的叠加。当以在相关波数范围 内无吸收的 $\mathrm{CCl}_{4}$ 稀释叔丁醇时, 其理想光谱将随摩 尔分数而线性变化。然而, 实际情况并非如此, 溶液中各物种的数量的变化将是非线性的。实际 光谱减去理想光谱, 将会出现正峰和负峰, 分别 代表相对数量增加和减少了的物种。这里说相对
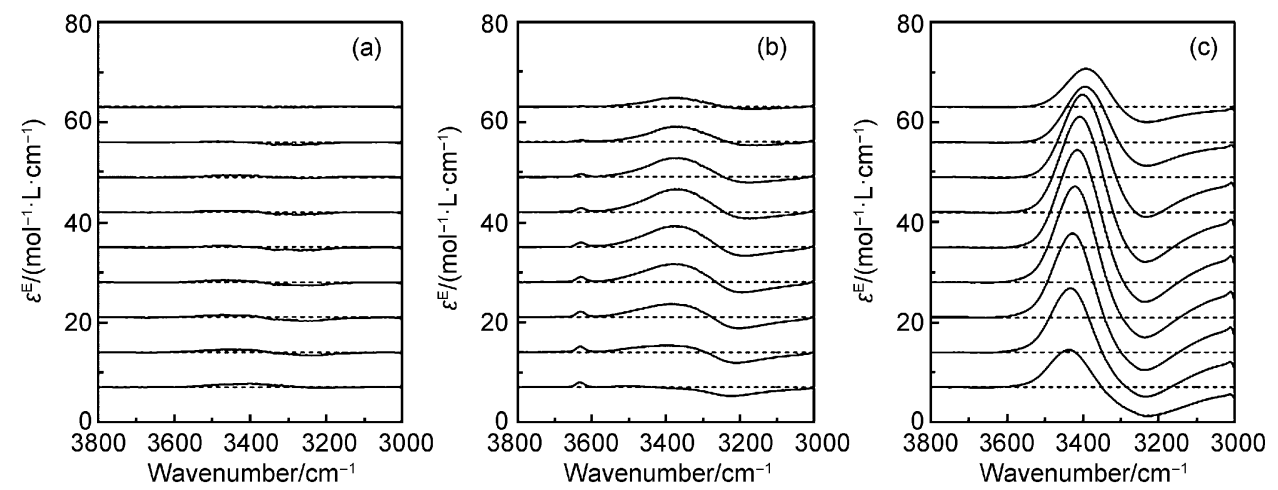

图2 O-H伸缩振动的超额红外光谱 ${ }^{13}$

Fig.2 Excess infrared spectra in the $\mathrm{O}-\mathrm{H}$ stretching vibration region ${ }^{13}$

(a) ethanol-methanol; (b) ethanol- $\mathrm{CCl}_{4}$; (c) ethanol-DMSO system. $\varepsilon$ : molar absorption coefficient; ${ }^{\mathrm{E}}$ : excess absorption coefficient 

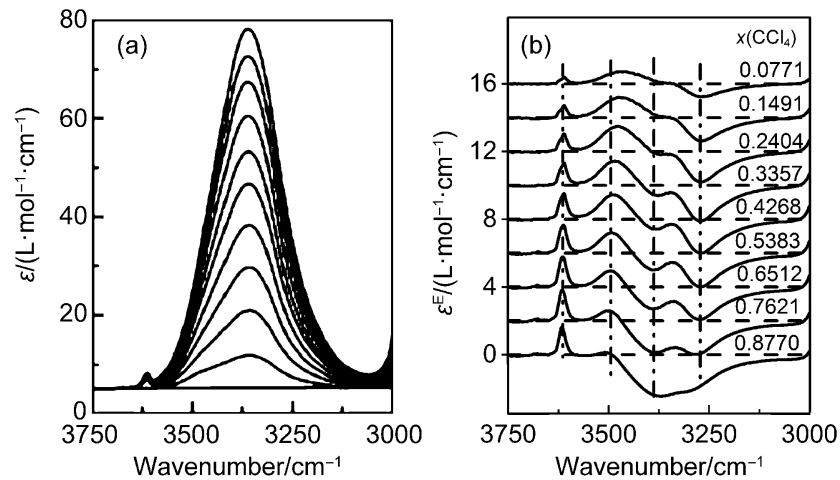

图3 叔丁醇-四氯化碳体系中 $O-H$ 伸缩振动的红外光谱(a)和超额红外光谱(b) ${ }^{13}$

Fig.3 Infrared spectra (a) and excess infrared spectra (b) of the $\mathrm{O}-\mathrm{H}$ stretching vibration in tert-butanol-CCl${ }_{4} \mathbf{S y s t e m}^{13}$

数量, 是因为我们定义的超额光谱以摩尔吸光度 为函数, 其物理意义是单位光程下单位浓度的光 谱。此外还需要说明的是, 一个基团的不同存在 形式相当于不同的化学环境, 存在形式的数目可 以是少数几个, 也可以是无穷多个。前者对应于 分子之间存在特殊强相互作用的情况, 若分辨率 好, 可以观察到若干位置相对固定的正峰和负 峰; 后者对应于简单的溶剂化作用或者分子构型 构象的调整和电荷分布的变化, 观察到的超额峰 位置不固定, 若分辨率不是很高, 将只可观察到 正峰或者负峰。

有了这样的认识, 图3(b)叔丁醇-四氯化碳体 系的超额红外光谱就很容易解读了: 图中自左至 右出现了两个正峰和两个负峰, 分别被归属为叔 丁醇的单体、二聚体、三聚体和多聚体。前二者 为正后二者为负表明, 随四氯化碳的加入, 单体 和二聚体的比例在增加, 而三聚体和多聚体的比 例在减少。很显然, 超额红外光谱提供了比普通 光谱更丰富的信息。

\section{3 超额峰的正负号提供亚分子层次结构信息}

在超额红外光谱中, 如果相同基团同一振动 模式的超额峰出现了反号, 则说明这两个基团在 混合过程中发生了截然相反的变化。李庆忠等 ${ }^{12}$ 以 DMSO和甲醇二元体系为例, 利用超额红外光谱研 究了“后方集团”烷基在氢键形成过程中的作用。在 DMSO和甲醇体系的红外光谱中，甲醇的甲基摇摆 振动的吸收峰位置在 $1115 \mathrm{~cm}^{-1}$ 附近, 而DMSO分子 中甲基摇摆振动的吸收峰位置在 $952 \mathrm{~cm}^{-1}$ 。他们发 现, 在红外光谱(图4(a))中, 只能看到随着各自浓 度的变化, 两个甲基摇摆振动的红外吸收强度相 应地单调变化。而在超额光谱(图4(b)) 中, 这两个
甲基的摇摆振动变化是明显不同的, DMSO分子的 甲基摇摆振动的超额光谱为正值，而甲醇分子的 甲基摇摆振动的超额光谱为负值。从正负峰结果 可以看出, DMSO和甲醇的甲基在形成氢键的过程 中发挥了不同的作用。量子化学计算表明, 这种 差异归因于甲基电荷密度变化引起的分子偶极的 变化。在甲醇-DMSO氢键作用体形成时, 甲醇中 甲基的电荷变化为负值, 即烷基上电子云密度增 大, 起吸电子作用; 而DMSO中电荷变化为正值, 即烷基电子云密度降低, 起给电子作用。它们均 对氢键的形成起到了积极的作用。核磁共振 (NMR)实验结果支持这个解释。

王楠楠 ${ }^{18}$ 等对 $N$-正丁基吡咯四氟嗍酸盐 ([bpy] $\left[\mathrm{BF}_{4}\right]$ )和DMSO体系进行了研究。在超额红外 光谱中, DMSO- $\mathrm{d}_{6}$ 的甲基和 $[\mathrm{bpy}]^{+}$的正丁基显示了 不同的光谱行为, 对应着形成 $[\mathrm{bpy}]^{+}-\mathrm{DMSO}$ 氢键作 用体时, DMSO的甲基可能起到给电子作用，而
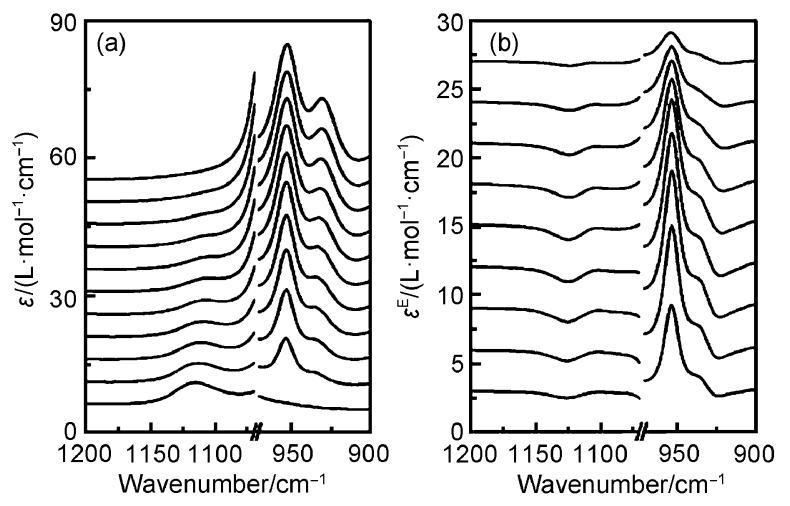

图4 DMSO和甲醇体系中甲基弯曲振动的红外光谱(a)和 超额红外光谱 $(b)^{12}$

Fig.4 Infrared spectra (a) and excess infrared spectra (b) of the $-\mathrm{CH}_{3}$ bending vibrations in DMSO-methanol system ${ }^{12}$ 
$[\mathrm{bpy}]^{+}$的正丁基可能起到得电子的作用。DMSO的 甲基和 $[\mathrm{bpy}]^{+}$的正丁基在形成氢键前后的NPA电荷 计算结果也证实了上面的预测。可见，超额光谱 正负峰可以反映出电子密度变化的信息, 这是普 通红外光谱所观察不到的, 再结合量子化学计算 的电荷变化的结果, 可以反映出亚分子层次分子 间相互作用的信息。

\section{4 判断分子间选择性相互作用的优先性}

王楠楠等 ${ }^{19}$ 在超额红外光谱定义的基础上, 定 义了超额摩尔吸光度表示的某基团的相对作用程 度的参数, 可以对分子间选择性相互作用的优先 性进行判断, 其推导如下:

由于吸光度具有加和性, 某一波数处的吸光 度可以表示为两组分的实际吸光度之和, 则公式 (6)在某一波数处的超额摩尔吸光度可以表示为:

$$
\begin{aligned}
\varepsilon^{\mathrm{E}} & =x_{1}\left(\frac{A_{1}}{d C_{1}}-\varepsilon_{1}^{*}\right)+x_{2}\left(\frac{A_{2}}{d C_{2}}-\varepsilon_{2}^{*}\right) \\
& =x_{1}\left(\varepsilon_{1}-\varepsilon_{1}^{*}\right)+x_{2}\left(\varepsilon_{2}-\varepsilon_{2}^{*}\right)
\end{aligned}
$$

式中, $\left(\varepsilon_{1}-\varepsilon_{1}^{*}\right)$ 代表组分 1 在混合物中表现出来的 摩尔吸光系数和纯组分 1 的摩尔吸光系数的差值, 称为摩尔吸光系数偏离值, 以 $\varepsilon_{1, \mathrm{~d}}$ 表示。同理 $\left(\varepsilon_{2}-\varepsilon_{2}^{*}\right)$ 可表示为 $\varepsilon_{2, \mathrm{~d}}$, 则超额摩尔吸光度可以表示 为:

$$
\varepsilon^{\mathrm{E}}=x_{1} \varepsilon_{1, \mathrm{~d}}+x_{2} \varepsilon_{2, \mathrm{~d}}
$$

当被讨论的吸收峰 $j$ 完全归属于某一组分时, 即此波数范围另一组分的吸光度为 0 , 则可以通过 超额摩尔吸光度得到一定浓度下的混合物中振动 方式 $j$ 的摩尔吸光系数偏离值:

$$
\varepsilon_{j, \mathrm{~d}}=\varepsilon^{\mathrm{E}} / x_{j}
$$

式中, $x_{j}$ 表示振动方式 $j$ 所在组分即目标组分的摩尔

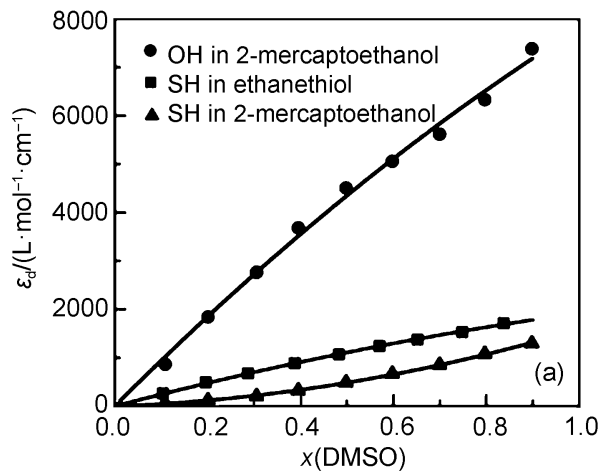

分数。

振动方式 $j$ 的摩尔吸光系数偏离值可反映该组 分与另一组分的相互作用程度，但不同基团摩尔 吸光系数的灵敏程度应不同。据此, 寻找参考点 做归一化处理, 定义相对作用程度参数如下:

$$
\eta_{j}=\frac{\varepsilon_{j, \mathrm{~d}}}{\varepsilon_{j, \mathrm{~d}, \mathrm{ref}}}=\frac{\varepsilon^{\mathrm{E}} / x_{j}}{\varepsilon_{\mathrm{ref}}^{\mathrm{E}} / x_{j, \mathrm{ref}}}
$$

式中, $\varepsilon_{\mathrm{ref}}^{\mathrm{E}}$ 和 $x_{j, \mathrm{ref}}$ 分别表示 $j$ 组分在参考点的超额摩 尔吸光度和摩尔分数。需要指出的是, 对于 $j$ 组分 的某一振动模式, 其超额摩尔吸光系数往往既含 有正值部分, 也含有负值部分, 因此采用该吸收 峰(或吸收带)的积分值更好。

作为选择性相互作用的一个例子, 王楠楠 等 ${ }^{19}$ 用本方法研究了颈基乙醇的羟基和颈基两个官 能团与DMSO形成氢键的先后顺序。图 5(a)为硫基 乙醇-DMSO体系中 $\mathrm{OH}$ 和 $\mathrm{SH}$ 伸缩振动的摩尔吸光系 数偏离值, 可见羟基比巯基的相关量变化要显著 得多。选择DMSO的摩尔分数为 0.9 的点作为参考 点, 根据公式(11), 可以得到相对作用程度参数 $\eta$, 如图5(b)所示。可以看到, 颈基乙醇中 $\mathrm{OH}$ 的相 对作用程度参数显著大于放 $\mathrm{SH}$ 的, 表明 $\mathrm{DMSO}$ 优 先与 $\mathrm{OH}$ 形成氢键。这里的逻辑推理可以参考贾奇 等 ${ }^{20}$ 的文章。作为对比, 王楠楠等还研究了乙硫醇DMSO体系, 所得到的相对作用程度参数与颈基乙 醇中 $\mathrm{OH}$ 的相对作用程度参数具有非常相近的变化 规律(图 5(b)), 表明分子中不存在 $\mathrm{OH}$ 时, $\mathrm{SH}$ 和 DMSO能够很好地发生氢键作用; 当分子中存在 $\mathrm{OH}$ 时, SH和DMSO的氢键作用被抑制了。也就是 说，当体系中同时存在 $\mathrm{OH}$ 和 $\mathrm{SH}$ 时, DMSO将优先 选择与 $\mathrm{OH}$ 作用。

\section{5 鉴定溶液中分子的存在形式}

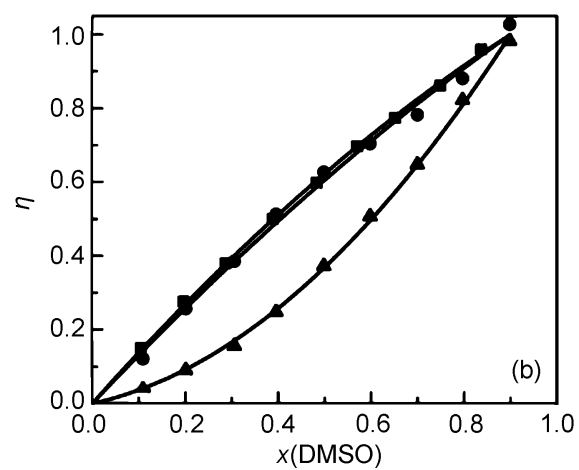

图5 摩尔吸光系数偏离值 $\varepsilon_{\mathrm{d}}(\mathrm{a})$; 相对作用程度参数 $\boldsymbol{\eta}(\mathrm{b})^{19}$

Fig.5 Integral values of $\varepsilon_{\mathrm{d}}$ (a) and a parameter on relative extent of interaction $\eta(b)^{19}$ $v(\mathrm{OH})$ and $v(\mathrm{SH})$ at different concentrations in 2-mercaptoethanol/ethanethiol-DMSO system. 
溶液结构是非常复杂的, 溶液中的分子可能 以多种形式存在, 如单体、同种分子的二聚体和 多聚体、异种分子间的作用体, 等等。超额光谱 的高灵敏度给我们提供了区分和鉴定分子不同存 在形式的可能。对于二组分溶液可以证明 ${ }^{21}$, 如果 超额光谱中正负超额峰的位置固定且零点的位置 也固定, 说明溶液中存在两种作用体。如果不是 全部固定, 则说明溶液中存在多种作用体(溶剂化 作用可以看作溶质有无穷多种存在形式)。对于只 有两种存在形式的情况, 证明过程如下。

对于一个二元体系 $M-S$ (其中 $M$ 为溶质, $S$ 为溶 剂), 假设 $\mathrm{S}$ 在溶质 $\mathrm{M}$ 的一个红外光谱吸收峰处没有 吸收。再假设溶质 $\mathrm{M}$ 在溶液中有两种作用体或存在 形式, 例如与溶剂有特殊作用和无特殊作用的两 种存在形式 $M_{1}$ 和 $M_{2}$, 它们的摩尔吸光系数分别 $\varepsilon_{21}$ 和 $\varepsilon_{22}$ 。这两种作用体的数量随浓度而变化, $\delta$ 表 示 $\mathrm{M}_{1}$ 占所有 $\mathrm{M}$ 的分数, 在纯溶质 $\mathrm{M}$ 中表示为 $\delta_{0}$ 。则 公式(5)可以表示为:

$$
\varepsilon^{\text {ideal }}=x_{2}\left[\delta_{0} \varepsilon_{21}+\left(1-\delta_{0}\right) \varepsilon_{22}\right]
$$

真实体系的摩尔吸光系数为:

$$
\varepsilon=x_{2}\left[\delta \varepsilon_{21}+(1-\delta) \varepsilon_{22}\right]
$$

则超额光谱可以表示为:

$$
\varepsilon^{\mathrm{E}}=x_{2}\left(\delta-\delta_{0}\right)\left(\varepsilon_{21}-\varepsilon_{22}\right)
$$

可见, 超额光谱的形状仅由 $\left(\varepsilon_{21}-\varepsilon_{22}\right)$ 决定, 当且 仅当 $\varepsilon_{21}=\varepsilon_{22}$ 时, 超额摩尔吸光度等于零。即在两 种作用体共存的二元体系中, 超额峰的位置和零 点的位置都是固定不变的。该结果也得到数字模 拟和实验结果的证实。

图6为 $\mathrm{C}_{6} \mathrm{~F}_{5} \mathrm{I}-$ cyclo- $\mathrm{C}_{6} \mathrm{H}_{12}$ 体系 $\mathrm{C}-\mathrm{I}$ 伸缩振动的红

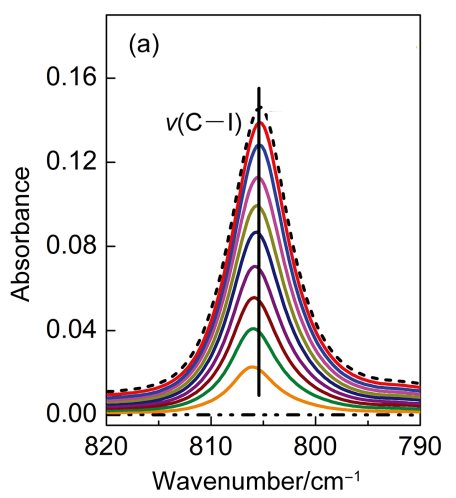

外光谱和超额光谱。在超额光谱中正负超额峰和 零点的位置都是固定的, 说明溶液中存在两种作 用体。由于在该体系中环己烷应是惰性溶剂, 这 两种作用体可能为碘代五氟苯的自聚体和单体。 结合量子化学计算, 低波数处负的超额峰指认为 来自碘代五氟苯二聚体, 而正的超额峰指认为碘 代五氟苯单体的吸收峰, 碘代五氟苯被环己烷稀 释的过程是其二聚体不断变为单体的过程。

\section{4 方法学研究进展}

\section{1 超额拉曼光谱}

刘世林课题组 ${ }^{14,15}$ 将超额光谱的概念应用于拉 曼光谱, 研究了盐对甲醇微观结构的影响和硝酸 镁 $\left(\mathrm{Mg}\left(\mathrm{NO}_{3}\right)_{2}\right)$ 溶液的离子缔合情况, 得到了一些有 意义的结果。

拉曼散射强度可以表示成如下形式 ${ }^{22}$ :

$$
I_{\mathrm{R}}=\left(I_{\mathrm{L}} \sigma k\right) d C
$$

其中 $I_{\mathrm{R}}$ 为所测的拉曼强度, $I_{\mathrm{L}}$ 为入射激光强度, $\sigma$ 为 绝对拉曼散射截面, $k$ 为测量常数, $d$ 为试样光路长 度, 而 $C$ 为浓度。由此可以得到, 拉曼散射强度正 比于浓度。当入射激光强度恒定(或进行归一化处 理), 对于指定的分子和相同的测量常数, 上式括 号中的量为常数, 定义其为摩尔拉曼散射系数 $\mu$, 单位 $\left(\mathrm{L} \cdot \mathrm{mol}^{-1} \cdot \mathrm{cm}^{-1}\right)$ 。与公式(2)相比, 可以看到这 里的摩尔拉曼散射系数类似于吸收光谱中的摩尔 吸光系数。对于理想溶液, 其摩尔拉曼散射系数 可用纯组分的摩尔散射系数的线性加和表示:

$$
\mu^{\text {ideal }}=x_{1} \mu_{1}^{*}+x_{2} \mu_{2}^{*}
$$

式中, $x_{1}$ 和 $x_{2}$ 分别表示混合体系中组分 1 和组分 2 的

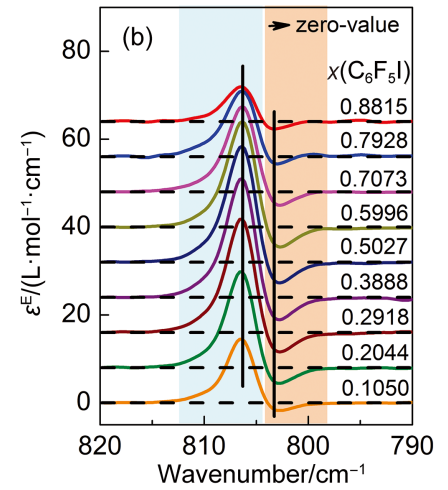

图6 碘代五氟苯和环己烷体系中 $C-I$ 伸缩振动的红外光谱(a)和超额红外光谱 $(b)^{21}$

Fig.6 Infrared spectra (a) and excess infrared spectra (b) of the $\mathrm{C}_{6} \mathrm{~F}_{5} I-c y c l o-\mathrm{C}_{6} \mathrm{H}_{12}$ system in the wavenumber range of the C-I stretching vibration ${ }^{21}$ 
摩尔分数, $\mu_{1}^{*}$ 和 $\mu_{2}^{*}$ 分别表示这两个纯组分的摩尔散 射系数, 可以通过测定纯物质的拉曼光谱得到。 在此基础上就可以仿照公式(1)-(6)进行超额拉曼光 谱的计算了。

在刘世林课题组的文章中 ${ }^{14,15}$, 超额拉曼光谱 用 $I^{\mathrm{E}}$ 表示, 与上式的关系是 $I^{\mathrm{E}}=\left(C_{1}+C_{2}\right) \mu^{\mathrm{E}}=$ $\left(C_{1}+C_{2}\right)\left(\mu-\mu^{\text {ideal }}\right)$ 。对于确定组成的溶液, 二 者的形状完全一样。

\section{2 超额偏摩尔红外光谱}

Koga等 ${ }^{23}-25$ 仿照偏摩尔热力学函数的思想, 在 超额红外光谱的基础上, 提出了超额偏摩尔吸光 度的概念. 某一组分 $j$ 的超额偏摩尔吸光度定义式如 下:

$$
\varepsilon_{j}^{\mathrm{E}}=n\left(\frac{\partial \varepsilon^{\mathrm{E}}}{\partial n_{j}}\right)
$$

式中, $n$ 表示体系中所有物质的总物质的量，对于 二组分体系, $n=n_{1}+n_{2}, n_{1}$ 和 $n_{2}$ 分别表示体系中 组分 1 和 2 的物质的量。组分 $j$ 的超额偏摩尔吸光度 的物理意义是该组分对超额摩尔吸光度的贡献。

Koga课题组 ${ }^{23-25}$ 用超额摩尔吸光度对多个水溶液体 系进行了研究。

\section{3 假二元固体超额红外光谱}

对于固体体系, 红外光谱测量要采用透射 法, 其核心的问题是光程难以准确测定。王楠楠 等 ${ }^{26}$ 采用内标法(IS) 来测定光程长度。他们以 $\mathrm{KSCN}$ 为内标, 研究了 Y 型分子篎和其包裹的锞离 子间主客体相互作用。内标的选择有几个准则: 内标须是惰性的, 不与待测样品发生特殊相互作 用; 内标中作为标准的吸收峰不能与待测样品的 吸收峰重叠。制备样品时, 内标的浓度应尽可能 小, 以保证Beer-Lambert定律的成立, 即标准物质 的摩尔吸光系数为常数。当然, 内标的吸收峰应 有足够的信噪比。以超额摩尔吸光度表示的超额 光谱计算公式如下:

$$
\begin{aligned}
\varepsilon^{\mathrm{E}}= & \varepsilon_{\mathrm{IS}} \frac{M_{1} M_{2}}{M_{\mathrm{IS}}\left(m_{1} M_{2}+m_{2} M_{1}\right)}\left[\frac{m_{\mathrm{IS}}}{A_{\mathrm{IS}}} A-\right. \\
& \left.\left(\frac{m_{1}}{m_{1}^{*}} \frac{m_{\mathrm{IS}, 1}^{*}}{A_{\mathrm{IS}, 1}^{*}} A_{1}^{*}+\frac{m_{2}}{m_{2}^{*}} \frac{m_{\mathrm{IS}, 2}^{*}}{A_{\mathrm{IS}, 2}^{*}} A_{2}^{*}\right)\right]
\end{aligned}
$$

式中, $m$ 为被研究的固体质量, $M$ 为该组分的相对 分子量, 1 和 2 分别为组分 1 和组分 $2, *$ 为纯组分。 从公式(18)可见, 内标物的摩尔吸光系数和分子量
并不影响超额光谱的基本形状(峰位置、零点位 置, 等等), 如果定义如下的相对超额吸光度, 则 在计算过程中无需知道内标的摩尔吸光系数, 可 以简化计算, 也有助于发掘新的内标物。

$$
\varepsilon_{\mathrm{R}}^{\mathrm{E}}=\frac{M_{\mathrm{IS}}}{\varepsilon_{\mathrm{IS}}} \varepsilon^{\mathrm{E}}
$$

如果固体样品的压片制备中还利用了 $\mathrm{KBr}$ ，而 关注的组分仍是两个, 这样的体系就成了假二元 体系。 $\mathrm{KBr}$ 和标准物质的存在或多或少会对两个关 注组分的相互作用产生影响, 因此在解释实验结 果时应予以适当考虑。

\section{5 应用举例}

超额光谱是分析分子间相互作用的有效手 段。超额光谱经过 10 年的发展, 内容越来越丰 富, 应用也越来越多, 已经成功地应用到了许多 体系中，从简单的小分子体系到较大的离子液体 体系等6,12-15,18,19,21,23-38。下面对超额光谱的部分应用进 行简单介绍。

\section{1 离子液体和共溶剂的相互作用}

张庆国 ${ }^{31,32}$ 、王楠楠 ${ }^{18}$ 、郑燕珍 ${ }^{6,33}$ 、何宏艳 ${ }^{34,35}$ 、 Kiefer ${ }^{36}$ 等对超额光谱与共溶剂的相互作用进行了 研究。以 $[\mathrm{BMIM}]\left[\mathrm{BF}_{4}\right]$ (1-丁基-3-甲基咪唑嗍酸盐)$\mathrm{CH}_{3} \mathrm{CN} / \mathrm{CD}_{3} \mathrm{CN}$ 体系为例进行简要的介绍, 图7为 [BMIM] $\left[\mathrm{BF}_{4}\right]-\mathrm{CH}_{3} \mathrm{CN} / \mathrm{CD}_{3} \mathrm{CN}$ 体系咪唑环 $\mathrm{C}-\mathrm{H}$ 伸缩 振动区域的红外光谱和超额红外光谱 ${ }^{6}$ 。超额光谱 中超额峰的个数可以帮助我们确定作用体的个 数, 咪唑环 $\mathrm{C} 2-\mathrm{H}$ 和 $\mathrm{C} 4,5-\mathrm{H}$ 伸缩振动区域都有三 种存在形式, 即在咪唑环 $\mathrm{C}-\mathrm{H}$ 伸缩振动区应该存 在六个超额峰, 分峰软件可以很容易地分出这六 个峰。通过超额红外光谱中超额峰的正负号可以 得到部分作用体归属的信息，正的超额峰代表新 作用体的生成或者原有作用体相对数量的增多, 负的超额峰代表原有作用体相对数量的减少。量 子化学计算进一步帮助我们完成对作用体的指 认。将量子化学计算得到的可能作用体的不同 $\mathrm{C} 2-\mathrm{H}$ 和 $\mathrm{C} 4,5-\mathrm{H}$ 伸缩振动的频率与分峰结果作 图，符合线性时，相关作用体的指认可信。

\section{2 盐溶液的微观结构}

刘世林 ${ }^{14,15}$ 和Koga $a^{24,25}$ 课题组分别利用超额拉曼 光谱、超额偏摩尔红外光谱对盐溶液的缔合行为 进行了分析。以室温下硝酸镁溶液的离子缔合情 

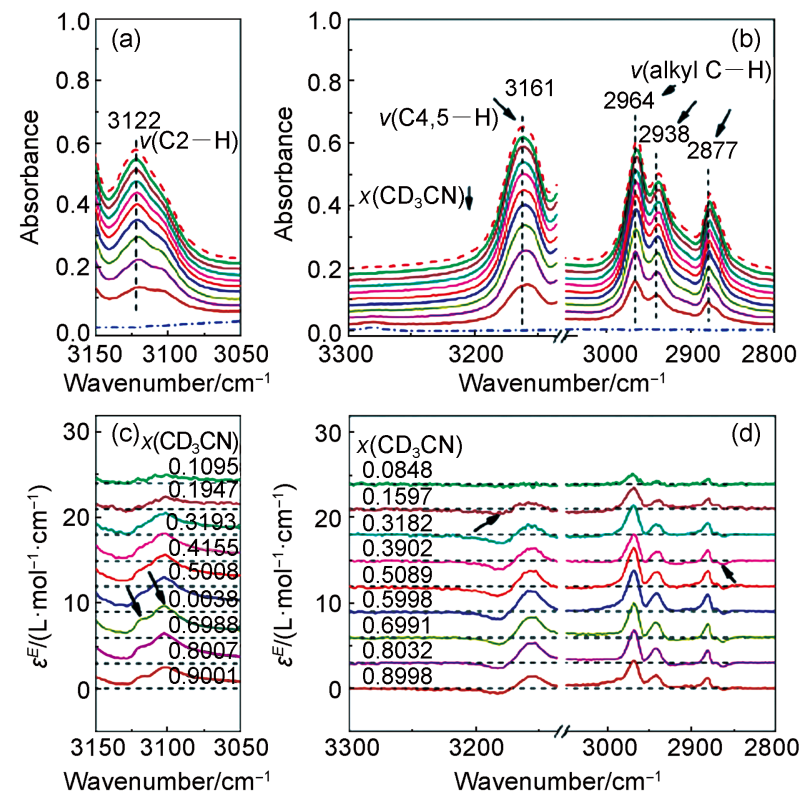

图7 红外光谱 $(\mathbf{a}, \mathbf{b})$ 和超额红外光谱 $(\mathbf{c}, \mathbf{d})^{\mathbf{6}}$

Fig.7 ATR-FTIR (a, b) and excess infrared $(c, d)$ spectra $^{6}$ $\mathrm{C} 2-\mathrm{H}$ stretching vibrations in $[\mathrm{BMIM}]\left[\mathrm{BF}_{4}\right]-\mathrm{CH}_{3} \mathrm{CN}$ system $(\mathrm{a}, \mathrm{c})$; $\mathrm{C} 4,5-\mathrm{H}$, alkyl $\mathrm{C}-\mathrm{H}$ stretching vibrationsin $[\mathrm{BMIM}]\left[\mathrm{BF}_{4}\right]-\mathrm{CD}_{3} \mathrm{CN}$ system $(b, d)$

况进行简要说明。王陈琛等 ${ }^{15}$ 分析了硝酸镁溶液羟 基伸缩振动区域和硝酸根全对称伸缩振动区域的 拉曼光谱和超额拉曼光谱。超额拉曼光谱结果显 示, 低浓度 $\left(<2.3 \mathrm{~mol} \cdot \mathrm{kg}^{-1}\right)$ 下, 阴离子第一水合层 的水分子含量随溶液浓度的升高呈线性增加, 在 较高浓度时 $\left(>2.3 \mathrm{~mol} \cdot \mathrm{kg}^{-1}\right)$, 该含量变化偏离了线 性关系, 这是因为硝酸镁溶液在高浓度时存在直 接接触离子对导致的。在硝酸根全对称伸缩振动 谱段除了直接接触离子对, 还观测到三种溶剂分 隔型离子对。对该区间内所有浓度的拉曼光谱和 超额光谱进行拟合, 给出了不同浓度下各种离子 对的相对含量的变化。

\section{3 卤键体系的溶液结构}

1983年Dumas提出了卤键的概念 39 。尽管关于 卤键研究的工作很多, 但目前的研究主要集中在 气体体系和量子化学计算中, 溶液体系较少 ${ }^{40-43}$ 。 超额光谱提供了一个新的研究溶液体系卤键的方 法。郑燕珍等 37,38 选择了 5 类氟苯衍生物研究它们与 DMSO间的相互作用, 对比了氢键与卤键的相互作 用的不同点。这五类氟苯衍生物分别为五氟苯 $\left(\mathrm{C}_{6} \mathrm{~F}_{5} \mathrm{H}\right)$ 、磑代五氟苯 $\left(\mathrm{C}_{6} \mathrm{~F}_{5} \mathrm{I}\right)$ 、溴代五氟苯 $\left(\mathrm{C}_{6} \mathrm{~F}_{5} \mathrm{Br}\right)$ 、氯代五氟苯 $\left(\mathrm{C}_{6} \mathrm{~F}_{5} \mathrm{Cl}\right)$ 和1-氯- $2,3,5,6$-四氟 苯 $\left(\mathrm{ClC}_{6} \mathrm{~F}_{4} \mathrm{H}\right)$ 。通过对 DMSO- $\mathrm{d}_{6}$ 中 $\mathrm{C}-\mathrm{D}$ 伸缩振动的
超额红外光谱的分析, 发现五个体系中负的超额 峰出现时DMSO的摩尔分数是不同的, 进而得到五 个体系中卤键(或氢键)的作用大小顺序: $\mathrm{C}_{6} \mathrm{~F}_{5} \mathrm{I}$ DMSO $>\mathrm{C}_{6} \mathrm{~F}_{5} \mathrm{H}-\mathrm{DMSO}>\mathrm{ClC}_{6} \mathrm{~F}_{4} \mathrm{H}-\mathrm{DMSO}>\mathrm{C}_{6} \mathrm{~F}_{5} \mathrm{Br}-$ $\mathrm{DMSO}>\mathrm{C}_{6} \mathrm{~F}_{5} \mathrm{Cl}-\mathrm{DMSO}$, 该结论得到量子化学计算 结果的支持。通过对 $\mathrm{C}-\mathrm{X}$ 键的振动光谱分析, 发 现在纯的氟苯衍生物中, $\mathrm{C}_{6} \mathrm{~F}_{5} \mathrm{Cl}$ 中可能只存在非聚 集的形式, 而 $\mathrm{C}_{6} \mathrm{~F}_{5} \mathrm{Br}$ 和 $\mathrm{C}_{6} \mathrm{~F}_{5}$ I中则可能存在单体和自 聚体的形式。

\section{6 总结与展望}

超额光谱是一个新的红外光谱分析手段、一 种研究分子间相互作用的有效方法。利用超额光 谱我们可以得到比原始光谱更加丰富的信息, 其 用途主要包括提高光谱表观分辨率、提供溶液结 构方面信息、判断二态性以及稳定缔合体的存 在、判断溶液的非理想性和判断分子间相互作用 优先性等。在过去十年中, 超额光谱经历了从无 到有, 且在方法学和应用上都有了很大的发展和 完善。在方法学上, 除超额红外光谱外, 还出现 了超额拉曼光谱、超额偏摩尔红外光谱。在应用 上, 超额光谱不仅已用于液体溶液体系, 也已用 于固体混合物的研究; 不仅已应用在小分子体 系, 也已用于离子液体等较大的分子体系。

超额光谱在研究分子间相互作用上体现了很 大的优势, 但是还存在一定的局限性。对于溶液 中分子存在形式的判断, 若只存在二态, 结论是 严格的; 若为多态, 结论不严格。对于超额峰正 负号的意义和规律还需要做更深入的研究工作。 目前超额光谱多用于二元体系, 对于三元以及更 复杂的体系尚缺少研究, 可以考虑假二元的方 法, 使超额光谱的应用范围更广。相信随着研究 内容的广度和深度的扩展, 更多的应用会被发 掘, 更多的分子间相互作用信息会被发现。

\section{References}

(1) Reichardt, C.; Welton, T. Solvents and Solvent Effects in Organic Chemistry, 4th ed.; Wiley-VCH Verlag \& Co. KGaA: Germany, Weinheim, 2011.

(2) Zhou, K. B.; Li, Y. D. Angew. Chem. Int. Edit. 2012, 51, 602. doi: 10.1002/anie.201102619

(3) Tang, F. Q.; Li, L. L.; Chen, D. Adv. Mater. 2012, 24, 1504. doi: 10.1002/adma.201104763

(4) Czarnecki, M. A.; Czarnik-Matusewicz, B.; Ozaki, Y.; Iwahashi, 
M. J. Phys. Chem. A 2000, 104, 4906. doi: 10.1021/jp991753e

(5) Fumino, K.; Wulf, A.; Ludwig, R. Angew. Chem. Int. Edit. 2008, 47, 8731. doi: 10.1002/anie.v47:45

(6) Zheng, Y. Z.; Wang, N. N.; Luo, J. J.; Zhou, Y.; Yu, Z. W. Phys. Chem. Chem. Phys. 2013, 15, 18055. doi: 10.1039/c3cp53356e

(7) Scatchard, G.; Hamer, W. J. J. Am. Chem. Soc. 1935, 57, 1805. doi: $10.1021 / \mathrm{ja} 01313 \mathrm{a} 016$

(8) Jiang, Y.; Liu, Y.; Sun, X. D.; Yu, Z. W. Thermochim. Acta 1991, 183, 99. doi: 10.1016/0040-6031(91)80449-S

(9) Yu, Z. W.; Liu, Y.; Sun, X. D. J. Solution Chem. 1992, 21, 497. doi: 10.1007/BF00649702

(10) Yu, Z. W.; Liu, Y.; Zhou, R.; Xue, F. Y. Sci. China Ser. B 2001, 44, 315. doi: 10.1007/BF02879622

(11) Zhao, X.; Yu, Z. W.; Zhou, R.; Liu, Y. J. Chem. Eng. Data 2001, 46, 1258. doi: $10.1021 / \mathrm{je} 0100600$

(12) Li, Q. Z.; Wu, G. S.; Yu, Z. W. J. Am. Chem. Soc. 2006, 128, 1438. doi: $10.1021 / \mathrm{ja} 0569149$

(13) Li, Q. Z.; Wang, N. N.; Zhou, Q.; Sun. S. Q.; Yu, Z. W. Appl. Spectrosc. 2008, 62, 166. doi: 10.1366/000370208783575663

(14) Yu, X. C.; Lin, K.; Hu, N. Y.; Zhou, X. G.; Liu, S. L. Acta Phys. -Chim. Sin. 2010, 26, 2473. [余小春, 林 珂, 胡乃银, 周 晓国, 刘世林. 物理化学学报, 2010, 26, 2473.] doi: 10.3866/PKU.WHXB20100922

(15) Wang, C. C.; Lin, K.; Hu, N. Y.; Zhou, X. G.; Liu, S. L. Acta Phys. -Chim. Sin. 2012, 28, 1823. [王陈琛, 林 珂, 胡乃银, 周 晓国, 刘世林. 物理化学学报, 2012, 28, 1823.] doi: 10.3866/PKU.WHXB201205154

(16) Weng, C. C. Fourier Transform Infrared Spectrometer; Chemical Industry Press: Beijing, 2005. [翁诗甫. 傅里叶变换红 外光谱仪. 北京: 化学工业出版社, 2005.]

(17) Hansen, W. N. Spectrochim. Acta 1965, 21, 815. doi: 10.1016/0371-1951(65)80039-X

(18) Wang, N. N.; Zhang, Q. G.; Wu, F. G.; Li, Q. Z.; Yu, Z. W J. Phys. Chem. B 2010, 114, 8689. doi: 10.1021/jp103438q

(19) Wang, N. N.; Jia, Q.; Li, Q. Z.; Yu, Z. W. J. Mol. Struct. 2008, $883-884,55$

(20) Jia, Q.; Wang, N. N.; Yu, Z. W. Appl. Spectrosc. 2009, 63, 344.

(21) Zhou, Y.; Zheng, Y. Z.; Sun, H. Y.; Deng, G.; Yu, Z. W. Sci. Rep. 2015, 5, 16379. doi: 10.1038/srep16379

(22) Yang, X. G.; Wu, Q. L. The Analysis and Application of Raman Spectroscopy; National Defence of Industry Press: Beijing, 2008. [杨绪刚, 吴琪琳. 拉曼光谱的分析与应用. 北京: 国防工业出 版社, 2008.]

(23) Koga, Y.; Sebe, F.; Minami, T.; Otake, K.; Saitow, K. I.;
Nishikawa, K. J. Phys. Chem. B 2009, 113, 11928.

(24) Sebe, F.; Nishikawa, K.; Koga, Y. Phys. Chem. Chem. Phys. 2012, 14, 4433. doi: 10.1039/c2cp23255c

(25) Sebe, F.; Nishikawa, K.; Koga, Y. J. Solution Chem. 2015, 44, 1833. doi: 10.1007/s10953-015-0376-3

(26) Wang, N. N.; Wang, Y.; Cheng, H. F.; Tao, Z.; Wang, J.; Wu, W. Z. $R S C A d v$. 2013, 3, 20237. doi: $10.1039 / \mathrm{c} 3 \mathrm{ra} 42634 \mathrm{c}$

(27) Wang, N. N.; Li, Q. Z.; Yu, Z. W. Appl. Spectrosc. 2009, 63, 1356. doi: 10.1366/000370209790109049

(28) Zhou, Y.; Zheng, Y. Z.; Sun, H. Y., Deng, G.; Yu, Z. W. J. Mol Struct. 2014, 1069, 251. doi: 10.1016/j.molstruc.2014.02.027

(29) Tong, H. J.; Yu, J. Y.; Zhang, Y. H.; Reid, J. P. J. Phys. Chem. A 2010, 114, 6795. doi: 10.1021/jp912180d

(30) Corsetti, S.; Zehentbauer, F. M.; McGloin, D.; Kiefer, J. Fuel 2015, 141, 136. doi: 10.1016/j.fuel.2014.10.025

(31) Zhang, Q. G.; Wang, N. N.; Yu, Z. W. J. Phys. Chem. B 2010, 114, 4747. doi: 10.1021/jp1009498

(32) Zhang, Q. G.; Wang, N. N.; Wang, S. L.; Yu, Z. W. J. Phys. Chem. B 2011, 115, 11127. doi: 10.1021/jp204305g

(33) Zheng, Y. Z.; He, H. Y.; Zhou, Y.; Yu, Z. W. J. Mol. Struct. 2014, 1069, 140. doi: 10.1016/j.molstruc.2014.01.013

(34) He, H. Y.; Chen, H.; Zheng, Y. Z.; Zhang, X. C.; Yao, X. Q.; Yu, Z. W.; Zhang, S. J. Aust. J. Chem. 2013, 66, 50. doi: $10.1071 / \mathrm{CH} 12308$

(35) He, H. Y.; Chen, H.; Zheng, Y. Z.; Zhang, S. J.; Yu, Z. W. Chem. Eng. Sci. 2015, 121, 169. doi: 10.1016/j.ces.2014.07.024

(36) Kiefer, J.; Molina, M. M.; Noack, K. ChemPhysChem 2012, 13, 1213. doi: 10.1002/cphc.v13.5

(37) Zheng, Y. Z.; Wang, N, N.; Zhou, Y.; Sun, H. Y.; Yu, Z. W. Phys. Chem. Chem. Phys. 2014, 16, 6946. doi: $10.1039 / \mathrm{c} 3 \mathrm{cp} 55451 \mathrm{a}$

(38) Zheng, Y. Z.; Deng, G.; Zhou, Y.; Sun, H. Y.; Yu, Z. W. ChemPhysChem 2015, 16, 2594. doi: 10.1002/cphc.v16.12

(39) Dumas, J. M.; Gomel, M.; Guerin, M. Halides, Pseudo-Halides and Azides 1983, 2, 985.

(40) Clark, T.; Hennemann, M.; Murray, J. S.; Politzer, P. J. Mol. Model. 2007, 13, 291. doi: 10.1007/s00894-006-0130-2

(41) Politzer, P.; Murray, J. S. ChemPhysChem 2013, 14, 278. doi: 10.1002/cphc. 201200799

(42) Politzer, P.; Murray, J. S.; Clark, T. Phys. Chem. Chem. Phys. 2010, 12, 7748. doi: 10.1039/c004189k

(43) Metrangolo, P.; Resnati, G.; Pilati, T.; Biella, S. Halogen Bonding in Crystal Engineering; Springer: Berlin Heidelberg, 2008. 